\title{
Assessment of emotional responses in preschoolers to an age-adapted stress task: Picture-based stress test and facial coding.
}

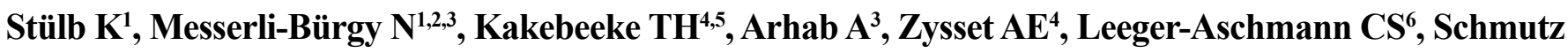 \\ EA $^{6}$, Meyer AH ${ }^{1,7}$, Bechtler B ${ }^{1}$, Schneider $S^{9}$, Kriemler $\mathbf{S}^{6 \#}$, Jenni OG ${ }^{4,5 \#}$, Puder JJ ${ }^{3,8 \#}$, Munsch $\mathbf{S}^{1^{* \#}}$ \\ ${ }^{1}$ Department of Clinical Psychology and Psychotherapy, University of Fribourg, 1700 Fribourg, Switzerland \\ ${ }^{2}$ Department of Psychology - Clinical Child Psychology and Biological Psychology, University of Fribourg, 1700 \\ Fribourg, Switzerland \\ ${ }^{3}$ Service of Endocrinology, Diabetes \& Metabolism, Lausanne University Hospital, 1011 Lausanne, Switzerland \\ ${ }^{4}$ Child Development Center, University Children's Hospital Zurich, 8032 Zurich, Switzerland \\ ${ }^{5}$ Children's Research Center, University Children's Hospital Zurich, 8032 Zurich, Switzerland \\ ${ }^{6}$ Epidemiology, Biostatistics and Prevention Institute, University of Zurich, 8001 Zurich, Switzerland \\ ${ }^{7}$ Department for Psychology, University of Basel, 4055 Basel, Switzerland \\ ${ }^{8}$ Service of Pediatric Endocrinology, Diabetology and Obesity, Lausanne University Hospital, 1011 Lausanne, \\ Switzerland \\ ${ }^{9}$ Faculty of Psychology, Clinical Child and Adolescent Psychology, Ruhr-Universität Bochum, 44787 Bochum, \\ Germany \\ \#Authors share last authorship
}

\begin{abstract}
Objective: Acute stress exposure leads to physiological, behavioral and emotional stress responses. While measurements of physiological stress responses in young children have improved, assessments of correlates of emotional responses to stress in children at preschool age are still limited to the coding of video-taped responses and their validity is questionable. Therefore, the aim of this study was to develop and investigate a method to assess correlates of emotional responses to stress by experienced emotional responses (Picture-Stress-Test, PST) and by facial expressed emotional responses.
\end{abstract}

Method: A total of 295 children aged 2-6 years of the SPLASHY study were assessed during an age-adapted stress task by heart rate variability, by videotapes (coded for positive and negative facial expression) and by the assessment of experienced emotional states at baseline, during the stress task and after the stress task. For this purpose, children were asked to estimate their own emotional state and to choose one of five pictures of rabbits with different emotional states (positive (happy) or negative (angry, anxious, stressed and sad)) depending on the estimated subjective emotional state.

Results: Both correlates of emotional responses and heart rate variability significantly changed under stress condition. Children showed lower levels of positive states in the PST and in the videotaped material during the stress task.

Conclusion: Measures of experienced emotional responses and of facial expression responses might represent valuable methods that could be combined to catch the broad range of correlates of emotional responses to a stress task in preschool children.

Keywords: SPLASHY, Child, Experienced emotional response, Emotional state assessment, PictureStress-Test (PST), Facial expression.

\section{Introduction}

The capacity to respond and adapt to challenging conditions is known to impact on behavioral problems even in young children. Previous studies revealed that limited flexibility to adapt and respond to stress or challenging conditions is related to externalizing behavior problems already in 1.5 to 3 -year old [1], but also to internalizing problems in older children aged 3 to 6 years [2].

Stress responses represent the adaptation to a challenging condition on a physiological, a cognitive, an emotional and a behavioral level. The assessment of stress response helps to understand potential risk conditions of psychopathological processes in young children. So far, mainly physiological stress responses have been in the focus of the research field and not emotional or behavioral responses to stress. Physiological stress responses (responses of the Hypothalamic-Pituitary-Adrenal axis (HPA) and the Autonomic Nervous System (ANS)) are commonly measured during lab-based stress tasks [3] such as the classic Trier Social Stress Test TSST [4] which is a valid 
and well-established social stress task for adults and adapted for older children [5]. The TSST includes socio-evaluative threats and uncontrollability. These factors are known to provoke physiological stress responses (HPA and ANS) even in young children [6-10].

However, exclusive assessment of physiological responses results in an incomplete picture [11] of stress regulation capacity as physiological and emotional response do not correspond regularly. Nevertheless, a coherent response has been assumed for many years [12] due to the shared underlying pathways of the limbic connections which initiate the emotional responses and the physiological response to stress of the HPA axis [13]. The dissociation of physiological and emotional stress responses has been underlined by a recent study of Campbell and Ehlert [11] showing only low to moderate correlations $(\mathrm{r}=0.3-0.5)$, but both responses being provoked by the classical or slightly modified TSST stress paradigm.

Correlates of emotional responses to stress are mostly assessed by self-reported subjective stress ratings during or after the TSST [11], but this assessment technique is limited to older children and adults. It cannot be used for younger children due to immature cognitive capacities of preschool children and limited self-introspection and verbalization. Two studies with preschoolers investigated correlates of emotional responses to an age-adapted version of the TSST by coding facial expression of positive and negative emotions based on video-recordings of the children during the stress task [7,10]. Both observed an increase of expressed negative emotions and a reduction of expressed positive emotions during the stress task in preschool children $[7,10]$. However, coded facial expression might reflect an immediate response of the individual distress level under acute stress conditions and therefore might rather represent a single dimension of these correlates of emotional response to stress. Further, a valid application of facial coding systems requires intense and time-consuming training of raters and it is unclear, whether interrater reliability remains sufficient if applied by other researchers than those of the original group.

In contrast, self-report assessments are likely to reflect experienced emotional states after initial processing [14-16]. The child's subjective perspective adds to an integrative picture of distinct facets of stress responses on an emotional level. Current self-reports of emotional distress due to stress exposure such as the Positive and Negative Affect Schedule for Children (PANAS) or the Acute Stress Checklist for Children (ASCKids) have been designed for older children of the $4^{\text {th }}$ to $8^{\text {th }}$ grade (PANAS-C; $\mathrm{m}=11.64$ years, $\mathrm{SD}=1.48$ ) and for children aged 8 to 17 years (ASC-Kids) [17,18]. Unfortunately, none of these assessment tools can be used to assess correlates of emotional responses in preschoolers due to their current immature cognitive capacity at that age.

Previous studies in clinical child psychology have shown that picture-based assessments match the preschoolers' skills to assess their own emotional state with satisfying reliability and validity such as in anxiety disorders [19-21] and in children with complex trauma [22]. Therefore, aiming to capture the broad picture of correlates of emotional stress responses, we developed a picture-based assessment to identify experienced emotional states of preschoolers during an age-adapted version of a TSST.

We hypothesized that correlates of emotional responses (experienced emotional state and facial expression) change during an age-adapted stress paradigm [23]. We expected higher levels of positive emotions (as expressed by the choice of happy rabbits and positive facial expressions in the video-recordings) than negative emotions during the baseline period (before the task when children do not know about a challenging task). Further we expected a reduction of positive emotions (less choices of happy rabbit and less positive facial expressions) and an increase of negative emotions during the exposure to the stress task, and a physiological stress response during the stress task (detected by changes of heart rate variability measures); and finally, a change back to high levels of positive emotions (more often choices of happy rabbits) and low levels of negative emotions after the stress task.

\section{Methods}

\section{Participants}

The current study was part of the second assessment time point (in 2015) one year after the start of the multi-site prospective cohort Swiss Preschoolers' health study SPLASHY in 2014, where 382 children aged 2-6 years were assessed (ISRCTN41045021). Details of the study design and the overall objectives have been previously described [23]. 351 children (m/f 184/167, mean age $=4.85(\mathrm{SD}=0.67))$ completed the stress task during the third testing afternoon in 2015 and provided data on experienced emotional responses, whereof video material was collected in 295 children and valid heart rate data in 219 children. The study was approved by the local ethical committees (No 338/13 for the Ethical Committee of the Canton of Vaud as the main ethical committee) and was in accordance with the Declaration of Helsinki. Parents provided written informed consent.

\section{Procedure}

The children completed an age-adapted stress task based on Kryski $[7,9,23]$ at the childcare centers. Our version of the stress task procedure included a baseline period, a stress task period and a recovery period. Within the stress task, children were told to complete a matching task under time pressure to win a previously chosen soft toy. Time was manipulated, and children were expected to perceive high levels of distress due to repeated failures and potential loss of an incentive [23]. After the third failure, the story of the task was dissolved and the children received their incentive and were informed that they did a great job. Assessment of responses to the stress task included emotional and physiological measures before the task (baseline), during (stress task) and after the stress task (recovery) as shown in Figure 1.

\section{Materials}

\section{Correlates of emotional responses}

Experienced emotional responses were assessed by the picturebased assessment "Picture-Stress-Test" (PST; [24]). The PST comprises cards with portrayed animals (rabbits) depicting five different emotional states (positive (happy) and negative (angry, anxious, stressed and sad)) and one rabbit with a neutral facial expression to explain the task (Figure 2). The child is first 


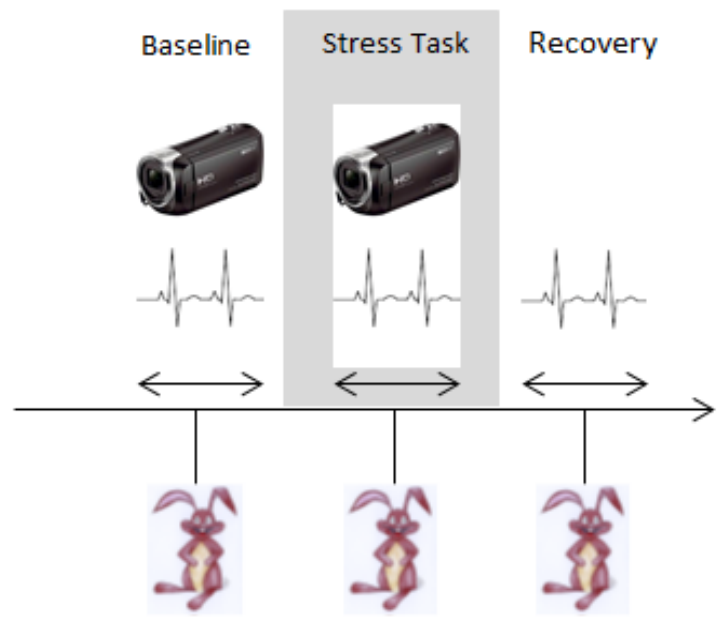

Figure 1. Assessment time points of emotional responses (experienced using the Picture Stress Test and facially expressed using video recording) and physiological responses (HRV measures) before (baseline), during the stress task (stress task) and after the task (recovery). Note: Recovery period was not video-taped.
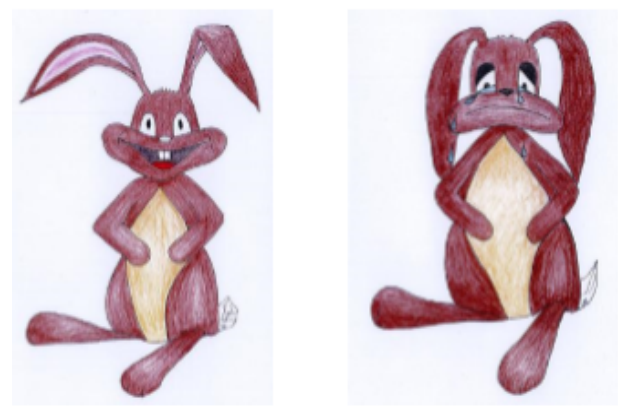

Figure 2. Examples of a positive (happy) and a negative emotional state (sad) of the Picture Stress Test (PST).

introduced to the assessment tool by a short story including the neutral rabbit, where the child is told that the neutral rabbit awaits a challenging task and perceives different feelings (showing the 5 rabbits with different emotional states). The child is then asked to identify all emotional states represented by the different rabbits. Emotional states that the child does not identify are explained by the tester. Finally, the child is asked to choose one of the emotional rabbits which best mimics the child's own current emotional state when awaiting a challenging task at baseline prior to the task. The choice of the rabbit picture with a specific emotional state is noted and is similarly reassessed at further time points during and after the stress task. In order to test whether coded facial expression of negative or positive emotions corresponded with the choice of the rabbits, children's choices were summarized in one variable reflecting rabbits with negative emotions (anxious, afraid, angry and stressed) and one with positive emotions (happy) similar to the video-coding strategy of facial expression data. Neutral rabbits are not considered for analyses.

Facial expressed emotional responses were assessed through video-taped material of the baseline and stress task condition. Video material was coded for a total of 294 children by trained coders. Facially expressed emotions were assessed on a Likert scale reflecting negative $(-3)$ to neutral $(0)$ and to positive emotions $(+3)$. Interrater reliability according to the ICC $(\mathrm{C}, 1)$
[25] was of $0.65(n=24)$ within a small group of three coders. When additional raters were included, even with an intensive training, single ICC $(\mathrm{C}, 1)$ was $0.59(\mathrm{n}=25)$.

\section{Physiological stress responses}

Heart rate variability (HRV) was measured using an eMotion HRV device (Mega Electronics, Kuopio, Finland) with a sampling frequency of $1000 \mathrm{~Hz}$ and accuracy of 1 millisecond. The HRV device was attached to the child's chest using disposable electrodes. Interbeat interval data was then extracted for 2 min time periods from heart rate recording at baseline, during the stress task and during recovery period (at each time in parallel to PST assessment). Data was exported to Kubios software (University of Eastern Finland, Kuopio, Finland) where outlier, artifact detection and smooth priors detrending $(\lambda=500)$ was performed and root mean square of successive difference (RMSSD) was calculated representing HRV during each time period.

\section{Statistical methods}

For the analysis of HRV we used a random intercept model with the difference between two time points (either baseline vs. stress task or stress task vs. recovery) as outcome, child as level-one and child care center as level-two variables. For the analysis of facial expressions, we used the same model as for HRV except that the outcome was not a difference score but the expressed emotion at the respective time point. Finally, for the analysis of emotional state (choosing positively or negatively connoted rabbits), we used a generalized linear mixed model with positive/negative rabbit as dichotomous outcome, time (baseline, stress test, recovery) as within-subjects predictor and the same level-one and two variables as for the other models.

\section{Result}

Analyses of the emotional states of these preschoolers revealed that children's emotional state clearly changed from baseline to the stress task and again from stress task to the period after the stress task. Thus, the probability to choose a positive rabbit decreased from baseline to the stress task from 92 to $72 \%$ (odds ratio $=0.24(95 \%-\mathrm{CI}=0.14-0.41), \mathrm{p}<0.001)$ and increased again from stress task to recovery period to $80 \%$ (odds ratio $=3.31$ (95\%-CI $=1.98-5.56), \mathrm{p}<0.001)$.

These results were in line with the results of the video-taped facial expression data, where children showed positive emotions during baseline since the average value was greater than the reference value 0 denoting neutral emotion $(b=0.23, \mathrm{SE}=0.05$, $\mathrm{p}<0.001, N=294)$ and clearly less positive emotions during the stress task $(b=-1.77, \mathrm{SE}=0.05, \mathrm{p}<0.001, N=285)$.

There was a clear decline in HRV during the age-adapted stress task as expected in response to a stress condition $(b=0.40$, $\mathrm{SE}=0.04, \mathrm{p}<0.001, N=219)$. During recovery, HRV increased again $(b=0.40, \mathrm{SE}=0.05, \mathrm{p}<0.001, N=216)$.

These findings are in line with our hypotheses that children experience less positive feelings during a stress task and more positive feelings before and again after the stress task and in parallel a change of HRV. 


\section{Discussion}

In line with previous studies using the same stress paradigm $[7,10]$, children in our sample showed an emotional and physiological response to the stress task. We found evidence for lower levels of positive emotions during the task than before and after the task. Interestingly, subjective experiences of emotional states in these young children (i.e. the choice of the rabbits in the PST) and video-taped emotional expressions both reflected a change to less positive emotions during the stress task. That means we were able to capture different correlates of emotional responses in preschoolers and were able to assess experienced emotional states for the first time in preschool children.

These results show that a picture-based stress task has a potential to identify subjective distress levels. Further, our expectation of parallel emotional and physiological responses to a stress task including changes of positive emotions and of HRV as previously shown in Kryski et al. [7] and Tolep and Dougherty [10], were fulfilled. Interestingly, a total of $68 \%$ of children kept choosing positive rabbits even during the stress task. We hypothesize that this might be due to immature cognitive capacities to identify a certain emotion in a picture, to compare it with the own perceived emotional state and to express this state by choosing a certain picture of a rabbit.

The picture quality or the fact that animal pictures were used, might have influenced the choice too as some emotions might not be detectable by children at a first stage. Our analyses on the identification of negative and positive rabbits based on 348 children revealed that $95 \%$ of children were able to identify the happy rabbit, $97 \%$ the sad rabbit, $93 \%$ the angry rabbit, $83 \%$ the anxious rabbit and $67 \%$ the stressed rabbit. We therefore conclude that picture quality was sufficient to choose pictures corresponding to positive or negative emotional states [24].

Although potentially valid data on correlates of emotional responses can be collected by PST and by facial coding of video-taped material, both techniques have their limitations. For instance, in this study, interrater reliability of coding facial expression material was only moderate and lower than in the studies of Kryski et al. [7] or Tolep and Dougherty [10]. Therefore, the results of facial expression coding need to be treated with caution. The physiological responses demonstrated that the age-adapted stress task was a sufficient stress exposure, but experiences in our research group clearly showed that even with intense training and repeated quality checks, interrater reliability for facial expression coding had its limitation. Facial expressions might have changed rapidly and could therefore not be reliably identified. However, the use of slow motion videotaping for coding revealed that strong emotion expressions did not occur in many children of this sample, which therefore limited identification of emotional responses in general and also led to limited variance in expressed emotion between children in this study. Thus, the relatively poor interrater reliability may be explained by the small between-subjects variability for facial expression in our sample which is well known to decrease ICC values, even if within-subjects (i.e. between rater-) variability is high. It should also be noted that the $\operatorname{ICC}(\mathrm{C}, 1)$ measure sensu McGraw and Wrong [25] was used in this study which is based on a single rater as the source of the actual measurement. The corresponding values for the ICC $(\mathrm{C}, \mathrm{k})$, i.e. based on multiple raters were 0.80 ( 3 raters, 23 children) and 0.79 (5 raters, 25 children) and thus comparable to those of Tolep and Dougherty [10] (0.77 and 0.82$)$, and only lower to those of Kryski et al. [7] (ICC of .91 and 0.96), but these two studies did not specify how they computed ICC. Furthermore, differences in coding instruments for facial expression between the studies and differences in the number of recoders used to assess ICC (other studies report one single reference person or did not define the number) of facial expression coding might also have influenced the observed discrepancies in ICC estimates between the different studies.

In comparison to the difficulties of facial expression coding, the use of the picture-based self-reports in children might therefore have advantages. In our study, we found that children at that age were able to identify emotional pictures and were able to perceive and compare their emotional state with those on the pictures. Therefore, their choice was likely to reflect a distinct perception and represent correlates of emotional responses. Nevertheless, the cognitive developmental stage of these children might have limited the reflection on the inner state and the comparison with a picture as well as the choice of pictures in the PST, as children at the age of 5 years still have immature emotion recognition [26]. Future studies should further compare whether different versions of pictures - rabbits versus children influence the reliability of choices. Additionally, it is important to further investigate children's capacities to express positive and negative emotional states [27] and their capability to reflect on the inner state and to their emotional states during the development in early, mid and late childhood.

\section{Conclusion}

Analyses of emotional responses to an age-adapted stress task in preschool children revealed that a picture-based test such as the PST and facial coding of video-taped material are both useful tools to assess children's correlates of emotional responses to a stress task. Children were able to identify emotional states on rabbit pictures and were able to change their choice of pictures according to their distress levels which was in line with changes of facially expressed emotions and changes of heart rate variability during the stress task. Nevertheless, both assessment types have their limitations: coding of facially expressed emotions depends explicitly on the intense training of a small number of raters and interrater reliability might be limited, whereas developmental levels of emotion recognition in children might impact on the choice of pictures in the picture-based stress test PST. Therefore, we assume that the combination of picture- and video-based assessments is needed to cover the broad picture of correlates of emotional responses in preschoolers. Future studies will need to further proof this combined assessment of emotional responses in this age group.

\section{Acknowledgment and Funding}

We would like to thank all children, families and day care centers that contributed data to SPLASHY. We also thank all students and the research team for their valuable contribution. The study was funded by a Sinergia grant from the SNF (Grant Number: CRSII3_147673) (http://p3.snf.ch/project-147673) and by the Jacobs Foundation. 


\section{Author's Contribution}

The overall study was designed and performed by SK, OJ, JP and SM. SM designed the overall aim and JP the physiological assessment of this stress sub-study. All authors conducted this research project and contributed to the data collection. AM assisted in statistical analyses. KS and NM drafted and NM and SM revised the first version of the manuscript. All co-authors elaborated and commented on the manuscript. All authors approved the final version of the manuscript. The last 4 authors have a shared last authorship.

\section{Competing Interests}

The authors declare that they have no competing interests.

\section{References}

1. Koss KJ, Mliner SB, Donzella B, et al. Early adversity, hypocortisolism, and behavior problems at school entry: A study of internationally adopted children. Psychoneuroendocrinology. 2016;66(3):31-8.

2. Laurent HK, Gilliam KS, Wright DB, et al. Child anxiety symptoms related to longitudinal cortisol trajectories and acute stress responses: Evidence of developmental stress sensitization. J Abnorm Psychol. 2015;124(1):68-79.

3. Kudielka BM, Wüst S. Human models in acute and chronic stress: Assessing determinants of individual hypothalamuspituitary-adrenal axis activity and reactivity. Stress. 2010;13(1):1-4.

4. Kudielka BM, Hellhammer DH, Kirschbaum C et al. Ten years of research with the Trier Social Stress Test -revisited. Social neuroscience: Integrating biological and psychological explanations of social behavior. Guilford Press New York, USA 2007;56:83.

5. Buske-Kirschbaum A, Jobst S, Wustmans A, et al. Attenuated free cortisol response to psychosocial stress in children with atopic dermatitis. Psychosom Med. 1997;59(4):419-26.

6. Dickerson SS, Kemeny ME. Acute stressors and cortisol responses: A theoretical integration and synthesis of laboratory research. Psychol Bull. 2004;130(3):355.

7. Kryski KR, Smith HJ, Sheikh HI, et al. Assessing stress reactivity indexed via salivary cortisol in preschool-aged children. Psychoneuroendocrino.2011;36(8):1127-36.

8. Roos LE, Giuliano RJ, Beauchamp KG, Gunnar M, Amidon B, Fisher PA. Validation of autonomic and endocrine reactivity to a laboratory stressor in young children. Psychoneuroendocrinology. 2017;77:51-5.

9. Stülb K, Messerli-Bürgy N, Kakebeeke TH, et al. Ageadapted stress task in preschoolers does not lead to uniform stress response. J Abnorm Child Psychol.

10. Tolep MR, Dougherty LR. The conundrum of the laboratory: Challenges of assessing preschool-age children's salivary cortisol reactivity. J Psychopathol Behav. 2014;36(3):350-7.

11. Campbell J, Ehlert U. Acute psychosocial stress: does the emotional stress response correspond with physiological responses? Psychoneuroendocrinology. 2012;37(8):1111-34.

12. Mauss IB, Levenson RW, McCarter L, et al. The tie that binds? Coherence among emotion experience, behavior, and physiology. Emotion. 2005;5(2):175.
13. Herman JP, Ostrander MM, Mueller NK, et al. Limbic system mechanisms of stress regulation: hypothalamopituitary-adrenocortical axis. Prog Neuropsychopharmacol Biol Psychiatry. 2005;29(8):1201-13.

14. Durbin CE. Validity of young children's self-reports of their emotion in response to structured laboratory tasks. Emotion. 2010;10(4):519.

15. Mauss IB, Robinson MD. Measures of emotion: A review. Cogn and Emot. 2009;23(2):209-37.

16. Reisenzein R, Studtmann M, Horstmann G. Coherence between emotion and facial expression: Evidence from laboratory experiments. Emot Rev. 2013;5(1):16-23.

17. Kassam-Adams N. The acute stress checklist for children (ASC-Kids): Development of a child self-report measure. J Trauma Stress. 2006;19(1):129-39.

18. Laurent J, Catanzaro SJ, Joiner Jr TE, et al. A measure of positive and negative affect for children: Scale development and preliminary validation. Psychol Assess. 1999;11(3):326.

19. Buchanan H, Niven $N$. Validation of a Facial Image Scale to assess child dental anxiety. Int J Paediatr Dent. 2002;12(1):47-52.

20. Dubi K, Schneider S. The Picture Anxiety Test (PAT): A new pictorial assessment of anxiety symptoms in young children. J Anxiety Disord. 2009;23(8):1148-57.

21. Venham LL, Gaulin-Kremer E. A self-report measure of situational anxiety for young children. Pediatr Dent. 1979;1(2):91-6.

22. King JA. Getting the Picture: A Cartoon-Based Assessment Tool for Complex Trauma in Children. Doctorate in Social Work (DSW) Dissertations. 2015; pp:68.

23. Messerli-Bürgy N, Kakebeeke TH, Arhab A, et al. The Swiss preschoolers health study (SPLASHY): Objectives and design of a prospective multi-site cohort study assessing psychological and physiological health in young children. BMC Paediatric. 2016;16(1):85.

24. Munsch S, Messerli-Bürgy N, Stülb K, et al. Picture Stress Test (PST).

25. McGraw KO, Wong SP. Forming inferences about some intraclass correlation coefficients. Psychol Method. 1996;1(1):30.

26. Rodger H, Vizioli, L, Ouyang, X, et al. Mapping the development of facial expression recognition. Developmental Sci. 2015;18(6):926-39.

27. Grossard C, Chaby L, Hun S, et al. Children facial expression production: Influence of age, gender, emotion subtype, elicitation condition and culture. Front Psychol. 2018;9:446.

\section{*Correspondence to:}

Munsch S

Department of Clinical Psychology and Psychotherapy University of Fribourg

Rue P.A. de Faucigny 2, 1700 Fribourg

Switzerland

Tel: +41263007657

E-mail: simone.munsch@unifr.ch 